\title{
NUEVOS DATOS SOBRE GALICISMOS DEL SIGLO XVIII
}

Para nuestro Estudio de galicismos en el español del siglo XVIII ${ }^{1}$ hemos elegido una de las novedades que ofrece el siglo, la prensa periódica, y ello nos ha permitido aportar nuevos datos sobre algunos galicismos.

Entre los objetivos de nuestro trabajo no estaba el detectar la fecha de primera documentación de los galicismos, pero al hilo de su desarrollo han ido apareciendo nuevas documentaciones y otros datos, como los referidos a la expansión y consolidación de algunos términos, que creemos pueden interesar a los estudiosos de la historia del léxico español.

Si bien hemos consultado una veintena de publicaciones periódicas, en un amplio muestreo de procedencias, fechas, etc., centramos fundamentalmente el trabajo en cuatro de ellas: La Gaceta de Madrid, de la que analizamos números correspondientes a los años 1751, 1761 y 1781, el Diario noticioso, curioso, erudito y comercial, público y económico, del que estudiamos números de los años 1758 y 1778, El Pensador (17621767) y El Censor (1781-1787).

Dada la falta de un diccionario histórico completo, para tratar de conocer el caudal léxico que entró a enriquecer la lengua en el siglo xviII hemos recurrido al magnífico Diccionario Crítico Etimológico Castellano

1 Tesis doctoral inédita presentada en la Universidad de Valladolid. Noviembre, 1982. 
e Hispánico, de J. Corominas y J. A. Pascual 2, y a otras variadas fuentes, como el trabajo "Galicismos", de B. Pottier ${ }^{3}$, Incorporaciones léxicas en el español del siglo XVIII, de G. Salvador ${ }^{4}$, los diccionarios del siglo, sobre todo el primer diccionario de la Academia y el Diccionario Castellano con las voces de Ciencias y Artes..., de Terreros y Pando 5, el trabajo de Baralt ${ }^{6}$, el Nuevo Diccionario Francés-Español de A. Capmany ${ }^{7}$. Por último, también hemos contado con numerosas fuentes indirectas, como el estudio de T. E. Hope sobre préstamos entre el francés y el italiano a lo largo de toda su historia, lo que nos ha permitido comprobar, por ejemplo, la coincidencia de numerosos galicismos del XVIII en italiano y español ${ }^{8}$, y algunas obras lexicográficas francesas ${ }^{9}$.

En el presente artículo ofrecemos datos sobre quince galicismos que pasamos a exponer siguiendo, por mayor comodidad, el orden alfabético.

\section{Arribo}

"Llegó a Sevilla [San Lauriano] y poco tiempo después de su arrivo murió Maximo su Arzobispo" (Diar. Not., 4 de julio de 1758).

"Al otro día de su arrivo la visitaron [a una dama] todos los caballeros del lugar» (Censor, Disc. XXIX).

«La mayor parte de las tropas de nuestro principal Exercito está ya en movimiento, y al arribo del Mariscal las hallará a todas prontas a obedecer sus ordenes» (Gac. de Mad., 14 de abril de 1761).

Del mismo modo que arribo, también hemos visto varias veces el verbo arribar 'llegar'. Este verbo, que, según $D C E C H$, se encuentra en castellano en textos antiguos y americanos con la acepción 'llegar en general', sorprendentemente es señalado como galicismo del siglo XvIII por Lapesa ${ }^{10}$.

En cuanto a arribo, que $D C E C H$ fecha h. 1800 (Jovellanos) remitiéndose al Diccionario de construcción..., de Cuervo, está en Autoridades: "Arribo. Lo mismo que llegada a alguna parte. Es voz moderna», y en

2 Citaremos DCECH. Todavía no ha aparecido el sexto y último volumen, por lo que para índices, etc., nos vemos obligados a consultar la primera edición (J. CoRominas, Diccionario Crítico Etimológico de la Lengua Castellana).

3 En Enciclopedia Lingüistica Hispánica, II, Madrid, 1967, págs. 127-151. Nos referimos a él con $E L H$.

4 Oviedo, 1973, Cuadernos de la Cátedra Feijoo.

5 Elaborados en 1723-1739 y 1786-1793, respectivamente. Citaremos Autoridades y Terreros.

6 R. Baralt, Diccionario de galicismos, Madrid, 1855, Imprenta Nacional.

7 Madrid, 1817, Imprenta de Sancha, 2.* ed.

8 Lexical Borrowing in the Romance Languages, Oxford, 1971.

9 Sobre todo Lexis, París, 1979, Larousse, y LITTRE, E., Dictionnaire de la langue française (1887), édition intégrale, 1971, Gallimard/Hachette.

10 R. LAPESA, Historia de la lengua española, Madrid, 1980, 8." ed., pág. 454. 
Terreros: "La llegada de alguno a esta, o la otra parte, puerto, fin de un negocio, etc.». Y las citas arriba aportadas dejan bien claro su significado 'llegada'.

Podría tratarse de un derivado formado naturalmente en castellano (Cuervo: «Etim. Nombre de acción sacado de arribar»), pero, puesto que es en el XVIII cuando aparece como neologismo, según Autoridades, pensamos en el fr. arrivée como modelo.

\section{Bandolín}

"Hay un sugeto, que enseña por cifra a tocar vihuela, bandurria, cythara, violin, tyorba, y bandolines de Italia" (Diar. Not., 18 de febrero de 1758).

En DCECH leemos: "Bandola 'cierto instrumento músico de cuatro cuerdas' [...] 1.a doc. Aut. [...] Es probable que el cast. bandola llegara a través del it. mandòla, que ya se halla a principios del XVII. El diminutivo de éste, mandolino, a través del fr. mandoline (s. XVIII), entró en fecha reciente en la forma mandolina o bandolina usual en muchas partes de América y en España misma [...] La forma tradicional que revistió el mismo vocablo grecolatino es bandurria. Deriv. Bandolín (princ. s. XIX)».

En Autoridades, bandola, y en Terreros: bandola y mandora. En los dos casos se diferencian bandola y bandurria, y no aparece ningún derivado del primero.

Si, como dice $D C E C H$, los derivados mandolina y bandolina entraron en nuestra lengua a partir del fr. mandoline, pensamos que también bandolín pudo ser un derivado que tomó como modelo la forma francesa. Además, Capmany traduce: «Mandoline. Bandolín: instrumento músico de cuerdas». El camino seguido por la palabra sería el siguiente: it. mandolino $>$ fr. mandoline $>$ esp. bandolin (luego bandolina). Y, como se aprecia por nuestra cita, podemos adelantar en medio siglo la fecha dada por el DCECH, resultando perfectamente coetáneos bandolín y el fr. mandoline (en 1759, según Lexis).

\section{Departamento}

En DCECH: "(Acad. ya 1817), del fr. département".

En Autoridades y Terreros encontramos departimiento 'división' como voz anticuada, lo mismo que el verbo departir 'dividir'. Efectivamente, con estas acepciones, según $D C E C H$, departir y departimiento se encuentran ya en Berceo.

Pero en el siglo xviı se toma la palabra del francés con unos significados nuevos a los que había llegado en aquella lengua. La Academia 
la recoge en 1817: «El distrito a que se extiende la jurisdicción o mando de cada capitán general o intendente de marina. Suele decirse por extensión de algunas otras divisiones de territorio, edificio, negociado".

Ya en nuestros textos del último cuarto del siglo xviII aparecen claros estos nuevos significados:

1: 'distrito a que se extiende la jurisdicción de cada capitán general o intendente de marina'. En la Gac. de Mad. de 6 de febrero de 1781 se habla de "departamentos de Marina".

2: 'sección de una oficina, negociado': "Señalo la [librería] de Esparza para lo tocante a Usos y Modas. Será del departamento de Maffeo todo lo concerniente a las costumbres...» (se refiere a los lugares donde debían enviar sus cartas los lectores según los temas de su contenido).

3: 'ministerio, ramo de la administración pública': «Departamento de la Guerra" (Gac. de Mad., 12 de enero de 1781), "Departamento de los negocios estrangeros» (Gac. de Mad., 9 de febrero de 1781), etc.

Hay que entender la aparición y expansión de departamento en el contexto de la importante reforma administrativa llevada a cabo por los Borbones, que supuso, en primer lugar, la sustitución del poder de la nobleza por una enérgica burocracia. Felipe $\mathrm{V}$ reglamentó los aspectos burocráticos, en su forma más completa y concreta, por la orden de 11 de julio de 1705 , que procura una adecuada distribución de competencias. Hay una Secretaría del Despacho Universal, que al principio se divide en dos secretarias: de Guerra por un lado, y de Hacienda y demás ramos, por otro. Luego, a lo largo del siglo, se van modificando el número y las competencias de cada secretaría, y así, por ejemplo, nos dice Autoridades: "Secretario del Despacho Universal [...] El día de oy está dividido este empleo entre quatro, el uno para lo tocante a Estado, y Ministros Extrangeros, otro para lo que pertenece a Gracia y Justicia, otro para la Guerra, y el otro para las dependencias de Hacienda, Indias y Marina».

Pues bien, esas Secretarías son las que también se denominan Departamentos, a pesar de que, como acabamos de ver, Academia (1817) no recoge explícitamente este significado. Tampoco Capmany en su traducción. Algunas veces, según hemos visto en textos citados por J. Beneyto "1, también se llaman Ministerios, y ya en el xIX Departamentos ministeriales. Pensamos que la aparición en nuestros textos de departamento y no ministerio se debe a la influencia francesa, teniendo en cuenta, además, que el término ministro, aunque también se utiliza, cada vez

11 Historia de la administración Española e Hispanoamericana, Madrid, 1958, Aguilar, pág. 531. 
más frecuentemente, como sinónimo de secretario, según dice A. Domínguez Ortiz ${ }^{12}$, no tiene todavía muy claramente el significado de un 'determinado y concreto empleo político o técnico' en Autoridades o Terreros.

$\mathrm{Y}$, ¿por qué con los otros sentidos si el español tenía división, distrito, etc.? El auge de la burocracia se aprecia en todos los niveles, y asi como la Secretaría de Despacho se dividía en departamentos, también lo hacía cualquier otra secretaría, o despacho u oficina.

\section{Devenir}

DCECH: "Devenir, raro y poco castizo (Acad. s. XIX), del fr. devenir; se emplea sustantivado ( $\mathrm{y}$ aun como verbo), en calidad de tecnicismo filosófico para traducir el alemán das Werden".

Hemos visto este verbo con el significado claro de 'hacerse, llegar a ser':

"Y la Justicia pide que tengamos un cuydado caritativo para los que sirviendo fielmente su Patria, han devenido incapaces de continuar el mismo servicio" (Suplemento al Extracto de Noticias Universales, 4 de marzo de 1728).

Ni Autoridades ni Terreros lo recogen. Capmany tampoco, y traduce el fr. devenir: "Empezar a ser una cosa diversa de lo que era: hacerse, ponerse, volverse diferente: pasar de un estado o condición a otra: convertirse, venir a parar, reducirse a otro predicamento".

La Academia lo registra en el xIX con estos significados, y además con el de 'suceder, acaecer', pero realmente el término es muy poco usado con cualquiera de ellos.

En definitiva, aunque lo hemos visto un siglo antes de que lo registrase Academia, no debía de estar todavía integrado en la lengua $-\mathrm{y}$ el que no lo utilice Capmany es muy indicativo-, pero la verdad es que, como verbo, nunca se integró, y, desde luego, nunca desplazó a las expresiones españolas convertirse, empezar a ser, hacerse, incluso cambiar, etcétera, para el primer significado, ni a sobrevenir, acontecer, etc., para el segundo.

Fagot

DCECH: «Fagot, tomado del fr. antic. fagot, íd., propiamente 'haz de leña', de origen desconocido; se le llama así por desmontarse en varias piezas, comparándolo a un conjunto de trozos de madera. 1.a doc.: Acad. 1843, no 1817».

12 Sociedad y estado en el siglo XVIII en España, Barcelona, 1976, Ariel, pág. 91. 
Hemos visto la forma fogot 'instrumento musical':

«Estas músicas, que suele haver en los templos, con flautas, fogot, o violines obligados, y en que se cantan sus concertadas arias de David Perez, del Duni, o de Galuppi» (El Pensador, Pens. XXVIII, pág. 9).

Por otro lado, Autoridades y Terreros registran fogote sólo como «Fagote, fagina, haz de leña menuda».

\section{Franela}

En DCECH: «Franela, del fr. flanelle y éste del ingl. flannel íd., [...]. 1. a doc.: Acad. 1817, no 1783; Pagés cita ej. de Bretón de los Herreros". $\mathrm{Y}$ en $E L H$ en el repertorio correspondiente a los siglos xIX y $\mathbf{x x}$.

Lo que nos resulta inexplicable, porque no sólo está en Terreros, con la forma flanela ("tela de lana mui fina, de que se hacen mantillas, y otras cosas. Tambien hai flanela de algodon y lana, de lana y pelo de cabra, y de lana y pelo de camello. Fr. Flanelle. Es comun en Francia, Inglaterra y España»), sino que ya aparece en su forma actual en un diario de 1758:

"Se perdio una Mantilla de franela» (Diar. Not., 4 de julio de 1758). Capmany todavía da la forma flanela.

\section{Libertinaje}

* Es cosa comun en las Señoritas recien casadas tomar un cierto atrevido modo de mirar, y un cierto libertinaje de locución a los primeros días de su boda" (Pensador, Pens. XXIX).

" $¿$ Merecería yo el nombre de padre si autorizase vuestro libertinage?» (Correo de los Ciegos, 17 de octubre de 1786).

En la primera cita se oponen esas maneras a las «modestas modales" que las señoritas tienen de solteras. La segunda pertenece a una "Carta de César" dirigida a los "celibatos de Roma», en la que les recrimina por preferir la soltería y la «entrega libre a las pasiones» a la legitimidad del matrimonio.

El adjetivo libertino todavía en Autoridades significa 'liberto', y dice $D C E C H$ que la acepción 'desenfrenado en lo moral' la tomó del francés en el siglo xIx. El fr. libertin ya en 1525 'indócil a la religión', y en el siglo XVII como 'inmoral' en general. Este es el mismo proceso que siguió también el it. libertino, según Hope.

En cuanto a libertinaje, $D C E C H$ lo incluye entre los derivados de libre, junto a libertino, pero no da ningún dato (ni origen, ni fechas). Creemos que el origen es, sin duda, el fr. libertinage, que se encuentra en el XVII con una amplia gama de significados: "Fantaisie, action de s'abandonner à ses penchants. 2. Indépendence, manque de soumission 
aux lois, aux règles. 3. Irréligion, impiété, incroyance» (Lexis). También el it. libertinaggio es para Hope un galicismo del XVIII.

Así pues, nos encontramos ante el hecho curioso de tener documentado libertinaje, tomado del francés, antes que la acepción 'desenfrenado en lo moral', también tomada del francés, para el propio libertino.

\section{Manteleta}

"Quien huviere perdido una Manteleta de griseta negra, que se halló en la Capilla de la Orden Tercera, acudirá al Tinte de sedas, calle Segovia...” (Diar. Not., 23 de febrero de 1758).

DCECH registra como galicismo mantelete, término de blasón, o de fortificación, o de vestido, y, efectivamente, esas son las acepciones que da Autoridades. Junto a él, entre los derivados de manto, y sin hacer ninguna referencia a su origen, está «manteleta (no Aut.; h. 1800, Jovellanos en Pagés, quien además cita un romance)». También en ELH está mantelete como tecnicismo, entre los galicismos de los siglos xVI y xVII.

Sin embargo, Terreros diferencia manteleta / mantelete: «Manteleta, una especie de adorno a modo de dengue, aunque por lo común más corto, que usan las señoras", y "Mantelete de obispo, manto o sotana...», "Mantelete en la Milicia, parapeto portátil de madera...», "Mantelete en el blasón, especie de manto menor que el Ducal».

En todo caso, aunque no hemos visto registrado el término como galicismo, pensamos que sí lo es. Ya se utilizaba a mediados de siglo como demuestran nuestra cita y el propio Terreros, y mantelete, que sí es considerado como tal, está fechado en $D C E C H$ sólo en 1725. Así que del fr. mantelet tendríamos en manteleta el significado «Petit manteau court, sans manches, que portent les femmes et qui couvre les épaules et les bras", y el resto, 'vestidura de prelado', 'parapeto de fortificación', etcétera, en mantelete.

Capmany traduce mantelet: "Muceta de obispo: Manteleta, capotillo de muger...».

\section{Minué}

Recogido como galicismo en $E L H$, pero en los siglos xix y xx (minuéminuete), y en DCECH (Minué, alguna vez minuete), que lo fecha en Academia 1817.

Sin embargo, es bastante frecuente en los periódicos analizados (formas minuet, minuete para el singular, minues para el plural):

"Minues figurados" (en un repertorio de bailes) (Diar. Not., 7 de febrero de 1758). 
"No se como ay quien se divierta en estos bayles. Yo me estoy durmiendo desde el primer minuete» (El Censor, Disc. XXIX), etc.

Y ya está en Terreros: "Minuete, especie de danza, cuyos tonos son prontos, y delicados [...]: hai variedad de minuetes, v. gr., del nardo, de trompas, de la mosca, etc.».

Capmany traduce: "Menuet. Minuette: cierto bayle de escuela».

También lo hemos visto con el significado 'composición musical' que había adquirido en francés:

«Seis Minues con el del cucu, á dos violines, por un profesor de esta Corte» (en los anuncios de venta de piezas musicales de la Gac. de Mad., 23 de febrero de 1781).

\section{Muselina}

Como galicismo en $E L H$ y $D C E C H$. Dice este último: "1. a doc.: moselina, Terrer.; mosulina, R. de la Cruz, fin del XvIII; muselina, Moratín, $\dagger$ 1828; Acad. 1803 [...] En castellano no es arabismo directo. Quizá lo sea el cat. mussolina, que es más antiguo...».

A esto queremos añadir que en Terreros hay tres formas: «Musolina, V. Mosulina». Luego ésta no aparece, pero sí moselina: «Lienzo de algodón mui fino y delicado. Fr. Mouseline». Y que en los periódicos hemos visto las formas musolina, varias veces, mosolina, una sola vez (en Diario de Valencia, 5 de julio de 1790), y la que sería definitiva, es decir, muselina:

«La urca Olandesa apresada [...] está cargada de muselinas, paños, cotonias y otros generos» (Gac. de Mad., 6 de marzo de 1781).

\section{Paquebot}

En DCECH: "paquebot o paquebote (-ot y -ote los emplea Fco. Palau, La vida de fray Junípero Serra, Méjico, 1787, y paquebotillo en pág. 10, y están ya en un diario marítimo de 1775, según me comunica el profesor Seán Page), adaptación del ingl. packboat íd., compuesto de pack 'paquete de cartas, etc.' + boat 'barco'».

A esto tenemos que replicar, en primer lugar, que es muy frecuente en la Gac. de Mad., por lo menos desde 1751:

«Ha tenido el Rey la noticia de haver entrado en la Bahía de Cádiz el dia 20 del passado el Paquebot nombrado Santa Elena» (Gac. de Mad., 2 de marzo de 1751).

«Ya se ha botado al agua una de las embarcaciones que deben sobstituir a los Paquebots, para assegurar nuestra correspondencia con el continente" (Gac. de Mad., 27 de enero de 1761).

«Los paquebotes-correos» (Gac. de Mad., 6 de febrero de 1781). 
«Varias cartas de Jamayca conducidas por el Paquebot Dashwood, confirman la pérdida del navío el Sterlingcastle» (Gac. de Mad., 23 de marzo de 1781), etc.

Nótese que las alternancias singular/plural son paquebot-paquebots (en los núms. de 1751 y 1761) y paquebot-paquebotes (en los de 1781).

$\mathrm{Y}$, en segundo lugar, que nos decidimos por incluirlo entre los galicismos atendiendo a que las formas halladas son siempre paquebot, paquebots, paquebotes, semejantes a la forma francesa en su grafía (el fr. paquebot desde mediados del xvir; en 1643 paquebouc), y porque Hope lo incluye también entre los galicismos que entraron en italiano en el XviII, reconociendo, por supuesto, como él hace, que "Ultimately Eng. packet-boat, but the intermediary role of Fr. paquebot is indisputable».

\section{Puf y polisón}

Si bien no hemos visto polisón en nuestros textos - sólo puf con el mismo significado-, queremos hacer algunas precisiones a lo apuntado por $D C E C H$.

"El método más útil de usar el Puf». Y en nota a pie de página: "Puf es una especic de almohadilla o caderilla que gastan las petimetras para alzar la ropa" (Diario de las Musas, 3 de diciembre de 1790).

Con este significado no lo hemos visto en ninguno de los diccionarios españoles (en realidad sólo lo recoge M. Moliner como 'asiento de tapicería en forma de taburete cilíndrico'). Creemos que se trata de la adopción en español del fr. pouf, que tiene su origen en la interjección onomatopéyica pouf! El sustantivo se documenta en 1829, según Lexis, y tiene los siguientes significados: "1. Siège bas, en cuir ou en tissu rembourré. 2. Tournure qui servait à faire bouffer la jupe parderrière. 3. Coiffure de femme sous Louis XVI", correspondiendo perfectamente el segundo de ellos a nuestra cita. La interjección pouf! expresa una explosión, o un soplo, un ruido sordo de caída..., y de ahí los matices de 'blando, inflado, abombado', que encontramos en los significados del sustantivo pouf, y en el verbo pouffer, cuyo primer significado era 'soplar', luego 'estallar en carcajadas', etc., semejante, por otro lado, a la onomatopeya en que se basa el ingl. puff -sustantivo y verbo-, 'soplo', 'soplar', etc. ${ }^{13}$.

13 En un sentido muy distinto tenemos otro derivado del fr. pouf: pufo "estafa, engaño, petardo', significado que no hemos visto para el francés pouf, pero sí para un puff que registra Littré: «Puff. Tromperie de charlatan, annonce pour leurrer», aunque no debía de ser muy corriente porque no está en ningún otro diccionario. 
Este accesorio femenino debió de estar de moda en el xvirI entre las petimetras, y el resultado de su uso se comenta en el Disc. XLIX del Censor: "también hincharon notablemente las partes posteriores, inferiores a su cintura", sin embargo la denominación puf no debió de usarse mucho, siendo sustituida por otro galicismo, polisón, que se documenta, según $D C E C H$, en Bretón de los Herreros, y que la Academia registra en 1925 .

Dice DCECH que polisón debe de ser un duplicado de polizón 'niño travieso', etc., "seguramente en el sentido de 'vestido inmodesto, travieso, liviano'». Pero nos parece una traslación demasiado complicada, y creemos que se trata sin más de un préstamo de significante (del fr. polisson) y de significado, puesto que también polisson significó esto mismo. Leemos en Littré: “ $\left(6 .^{\mathrm{a}} \mathrm{ac}\right.$.) Mouchoir empesé que les dames plaçaient audessous de la taille pour se donnerde l'épaisseur et de l'ampleur; on dit aujourd'hui tournure». Es decir, que el fr. polisson se tomó prestado dos veces en español: una en el siglo XVIII, polizón «el sugeto ocioso y sin destino, que anda de corrillo en corrillo" (Autoridades), y otra en el xIx, polisón 'almohadilla...'.

\section{Similor}

Como galicismo en DCECH (del fr. similor, compuesto híbrido del lat. similis y el fr. or), que da como 1.a documentación 1817.

Pero no sólo está ya en Terreros: "Similor, cierto metal amarillo que parece oro, y no es otra cosa que el Cink fundido con el cobre», sino que lo hemos visto con bastante frecuencia en los periódicos:

«El día siete del corriente echo de menos un sugeto una caxa de similor...» (Diar. Not., 14 de febrero de 1758).

«Una cadenita de similór» (Diar. Not., 21 de febrero de 1758), etc.

\section{Tisú}

Recogemos este término para corregir a $E L H$ que lo da entre los galicismos de los siglos xIx y Xx. Porque, como señala $D C E C H$, ya está en Autoridades («Tisú. Tela de seda mui doble bordada de flores varias sobre plata u oro, que passan desde el haz al embés. Es voz tomada del fr. Tissu, que vale texido. Llámase también Tesú»). Y en Terreros: «Tisú, tela de seda, y plata, u oro». Además, es frecuentísimo en los periódicos:

«Un chupetín de tisú, con campo verde, y ojaleado de oro» (Diar. Not., 16 de febrero de 1758).

«Un tocador a la francesa, con guarnición de tisú, y galón de oro» (Diar. Not., 23 de febrero de 1758), etc. 


\section{Viñeta}

En DCECH: «Viñeta (Acad. ya 1843), del fr. vignette 'adorno en figura de sarmientos que se pone en las primeras páginas de un libro'». ELH también lo incluye entre los galicismos de los siglos XIX y xx.

Sin embargo, no sólo está ya en Terreros: "Viñeta, llaman los impresores a la figura o labor que ponen al principio de un libro, o capítulo, y para adornar carteles, etc. Fr. Vignette [...] algunos la llaman en cast. viñuela V. Herrer. Dicc., pero los Impresores que dan aquí la ley, dicen viñeta, o ramo, o marmosete», sino que hemos visto el término en un diario de 1758:

«Grava assimismo, en madera, quanto se le pida para Marmosetos, Cabeceras, Viñetas, y Letras floridas de Imprenta" (Diar. Not., 1 de julio de 1758).

\section{Pilar Vaillejo Arróniz.}

